\title{
FATORES ASSOCIADOS AO TABAGISMO EM USUÁRIOS DA ESTRATÉGIA SAÚDE DA FAMÍLIA
}

\author{
Erildo Vicente Müller¹, Makcine Timm da Silva², Clóris Regina Blanski Grden³, Péricles Martim Reche \\ Pollyanna Kássia de Oliveira Borges ${ }^{4}$, Jacy Aurélia Vieira de Sousa ${ }^{3}$
}

\begin{abstract}
RESUMO: Objetivou-se identificar fatores associados ao tabagismo em usuários da estratégia saúde da família. Estudo epidemiológico transversal, descritivo e analítico. A amostra por conveniência compreendeu 100 indivíduos usuários de uma Unidade Saúde da Família de um município da região centro sul do Paraná. Houve predomínio das mulheres $(n=63 ; 63 \%)$, com média de idade de 49,5 anos, cor branca $(n=82 ; 82 \%)$, com ensino fundamental completo $(n=21 ; 21 \%)$ e casados $(n=49 ; 49 \%)$. O consumo médio diário de cigarro foi de 17,7 cigarros/ dia e 56 (56\%) participantes apresentavam grau de dependência muito elevado ou elevado à nicotina. Constatouse associação entre a média de tempo de tabagismo e idade $(p=0,001)$, estado civil viúvo $(p=0,035)$, colesterol elevado ( $p=0,035)$, uso de anti-hipertensivo $(p=0,031)$ e grau de dependência a nicotina muito elevada $(p=0,008)$. A identificação dos fatores associados ao tabagismo possibilita à equipe de saúde buscar e planejar estratégias de prevenção, tratamento e acompanhamento dos tabagistas.
\end{abstract}

DESCRITORES: Tabagismo; Hábito de fumar; Saúde da família; Tabaco; Estudos transversais.

\section{FACTORS ASSOCIATED WITH SMOKING AMONG USERS OF THE FAMILY HEALTH STRATEGY}

\begin{abstract}
The aim was to identify factors associated with smoking among users of the Family Health Strategy. A cross-sectional, descriptive and analytic epidemiological study. The sample by convenience encompassed 100 individuals who were users of a Family Health Unit from a city in the center-south region of the Brazilian State of Paraná. There was a predominance of women ( $n=63 ; 63 \%)$, with a mean age of 49.5 years, Caucasian $(n=82 ; 82 \%)$, who had completed junior high school $(n=21 ; 21 \%)$ and who were married $(n=49 ; 49 \%)$. Mean daily cigarette consumption was 17.7 cigarettes/day and $56(56 \%)$ participants had a very high or high degree of dependence on nicotine. An association was observed between mean smoking duration and age ( $p=0.001)$, marital status - widowed $(p=0.035)$, high cholesterol $(p=0.035)$, use of antihypertensives $(p=0.031)$ and very high degree of dependence on nicotine $(p=0.008)$. The identification of the factors associated with smoking makes it possible for the health team to seek and plan prevention strategies, treatment and monitoring of smokers.
\end{abstract}

DESCRIPTORS: Tobacco Use Disorder; Smoking; Family Health; Tobacco; Cross-sectional Studies.

\section{FACTORES ASOCIADOS AL TABAQUISMO EN USUARIOS DE LA ESTRATEGIA SALUD DE LA FAMILIA}

RESUMEN: Estudio cuyo propósito fue identificar factores asociados al tabaquismo en usuarios de la estrategia salud de la familia. Estudio epidemiológico transversal, descriptivo y analítico. El muestreo de conveniencia abarcó 100 individuos usuarios de una Unidad Salud de la Familia de un municipio de la región centro sur de Paraná. Hubo predominio de mujeres $(n=63 ; 63 \%)$, con edad media de 49,5 años, color blanco ( $n=82 ; 82 \%)$, con enseñanza básica completa $(n=21 ; 21 \%)$ y casados $(n=49 ; 49 \%)$. El consumo medio de cigarrillos fue de 17,7 al día; 56 (56\%) participantes presentaban grado de dependencia muy alto o alto de nicotina. Se constató asociación entre la media de tiempo de tabaquismo y la edad ( $p=0,001)$, estado civil viudo $(p=0,035)$, colesterol elevado ( $p=0,035$ ), uso de anti hipertensivo $(p=0,031)$ y grado de dependencia de nicotina muy alto $(p=0,008)$. La identificación de los factores asociados al tabaquismo posibilita al equipo de salud buscar y plantear estrategias de prevención, tratamiento y acompañamiento de los tabaquistas.

DESCRIPTORES: Tabaquismo; Hábito de fumar; Salud de la familia; Tabaco; Estudios transversales.

${ }^{1}$ Farmacêutico. Doutor em Saúde Coletiva. Docente de Enfermagem da Universidade Estadual de Ponta Grossa. Ponta Grossa, PR, Brasil.

${ }^{2}$ Enfermeira. Residente em urgência e emergência. Universidade Federal do Paraná. Curitiba, PR, Brasil.

${ }^{3}$ Enfermeira. Doutora em Enfermagem. Docente de Enfermagem da Universidade Estadual de Ponta Grossa. Ponta Grossa, PR, Brasil.

${ }^{4}$ Dentista. Doutora em Saúde Coletiva. Docente de Enfermagem da Universidade Estadual de Ponta Grossa. Ponta Grossa, PR, Brasil.

Autor Correspondente:

Recebido: 09/02/2017

Erildo Vicente Müller

Universidade Estadual de Ponta Grossa

Finalizado: $15 / 09 / 2017$

Av. General Carlos Cavalcanti, 4748 - 84030-900 - Ponta Grossa, PR, Brasil

E-mail: erildomuller@hotmail.com 


\section{INTRODUÇÃO}

O tabagismo é considerado a principal causa de morte evitável no mundo ${ }^{(1)}$ e no Brasil $^{(2)}$. Estimativas da Organização Mundial da Saúde (OMS) apontam que um terço da população mundial adulta, ou seja, 1 bilhão e 200 milhões de pessoas, são fumantes ${ }^{(1)}$.

Segundo a Pesquisa Nacional de Saúde ${ }^{(3)}$, a prevalência do uso atual de tabaco em pessoas na idade de 18 anos ou mais é de 15\%, com predomínio do sexo masculino $(19,2 \%)$ e da faixa etária de 40 a 59 anos (19,4\%). Quanto aos estados brasileiros, a região sul lidera o índice de usuários de tabaco e derivados, com destaque para o Paraná que registra $18,1 \%$ de fumantes ${ }^{(3)}$.

Inserido na Classificação Internacional de Doenças (CID-10) da OMS, o tabagismo é reconhecido como uma doença crônica ${ }^{(4)}$ que apresenta relação significativa com os principais grupos de doenças crônicas não transmissíveis (DCNT): doenças cardiovasculares, diabetes, neoplasias e doenças respiratórias crônicas ${ }^{(2)}$. Além disso, o hábito de fumar pode elevar o risco de morte em 20 a 30 vezes ${ }^{(1)}$, com estimativas de 200 mil mortes anuais decorrentes do tabagismo ${ }^{(5)}$.

O Brasil é considerado referência mundial no controle do tabagismo. Ações educativas, preventivas, terapêuticas e regulatórias contribuíram nas últimas décadas para a redução no hábito de fumar de $31,7 \%$ para $14,7 \%$ nos indivíduos adultos ${ }^{(5)}$. Contudo, o número de fumantes ainda é expressivo e com repercussões significativas no desenvolvimento de agravos à saúde, acarretando altos custos sociais e econômicos.

Nesse sentido, são relevantes ações individuais e coletivas com enfoque no tratamento e prevenção do tabagismo. A Atenção Básica $(\mathrm{AB})$, compreendida como o nível de atenção à saúde mais próximo do usuário, deve ser considerada o principal local de assistência ao usuário tabagista ${ }^{(6)}$. Nesse cenário, os profissionais de saúde, por meio do cuidado longitudinal, integral e próximo ao usuário, podem aumentar significativamente a efetividade das ações em saúde direcionadas a esta população ${ }^{(2)}$. Do mesmo modo, conhecer os fatores associados ao uso do tabaco oportuniza que a equipe de saúde planeje estratégias de prevenção, tratamento e acompanhamento dos tabagistas.

Diante do exposto, o objetivo do presente estudo foi identificar os fatores associados ao tabagismo em usuários da estratégia saúde da família.

\section{MÉTODO}

Trata-se de estudo epidemiológico do tipo transversal. A amostra, por conveniência, compreendeu 100 indivíduos usuários de uma Unidade de Saúde da Família de um município da região centro sul do estado do Paraná, a qual contempla duas equipes saúde da família e atende uma população de 8000 pessoas adscritas à área.

O campo de pesquisa foi selecionado porque os pesquisadores realizavam atividades do Programa de Educação pelo Trabalho para a Saúde, nas quais estavam compreendidos o diagnóstico de saúde da comunidade e posterior intervenção através de ações educativas (PET- Saúde).

Os critérios de inclusão foram: ser tabagista, morador da área de abrangência da Equipe Saúde da Família (ESF), estar disponível para entrevista no momento da visita domiciliar. Exclusão: menor de 18 anos, não aceitar participar do estudo e não estar no domicílio após duas visitas.

Os indivíduos incluídos no estudo foram selecionados por meio das fichas para cadastramento das famílias (Ficha A) dos Agentes Comunitárias de Saúde (ACS).

A coleta de dados foi realizada no período de fevereiro a maio de 2015, por meio de visitas domiciliares acompanhadas pelo Agente Comunitário de Saúde, com a utilização de formulário para o levantamento dos dados sociodemográficos. As variáveis de interesse pesquisadas foram: sexo, idade, cor da pele (autorreferida), escolaridade, estado civil e renda familiar. Foram investigados o consumo médio de cigarros e antecedentes familiares para doenças cardiovasculares. Avaliou-se o peso, altura, pressão arterial e realização de teste de glicemia. 
Para avaliar o grau de dependência da nicotina entre os fumantes, foi aplicado o teste denominado Fagerström Test for Nicotine Dependence (FTND) validado para utilização no Brasil ${ }^{(6)}$. O instrumento constitui-se de seis questões. A soma dos pontos resulta num escore, que varia de 0 a 10 , e indica o grau de dependência do fumante em: muito baixo, baixo, médio, elevado ou muito elevado, quando atingir, respectivamente, $0-2,3-4,5,6-7$ ou 8-10 pontos.

Para o cálculo do Índice de Massa Corpórea (IMC), foram determinados o peso e a altura. A verificação do peso deu-se por meio da balança eletrônica modelo EB9015 certificado pela SGS. Para verificar a altura, usou-se uma fita métrica simples de comprimento 1,50 m. Os critérios de classificação do IMC foram os preconizados pela Organização Mundial da Saúde $(\mathrm{OMS})^{(7)}$. Considerou-se desnutrido o sujeito com IMC $<18,5$, quando o IMC esteve entre 18,5 e 24,9 considerou-se o usuário com normalidade. Para valores de 25-29,9 ou $\geq 30$, os indivíduos foram classificados com sobrepeso e obesidade, respectivamente.

Para avaliação da circunferência abdominal, foi utilizada fita métrica, com o indivíduo na posição vertical foi circundada a cintura na linha do umbigo em um ponto médio entre a costela e a crista ilíaca, o critério de classificação adotado da International Diabetes Association (IDF) ${ }^{(8)}$. Os valores de referência para comparação entre normalidade e obesidade central foram, respectivamente, 80/90 centímetros para mulheres e 90/94 centímetros para os homens.

A pressão arterial foi aferida duas vezes pelo método indireto e técnica auscultatória com auxílio do esfigmomanômetro para adulto, aferido pelo Instituto Nacional de Metrologia, Qualidade e Tecnologia (IMETRO) em ambos os braços, com o sujeito na posição sentado e as pernas lado a lado. Os indivíduos foram classificados de acordo com as VII Diretrizes Brasileiras de Hipertensão Arterial ${ }^{(9)}$.

O teste de glicemia capilar foi realizado aproximadamente duas horas após o almoço ou café da manhã utilizando o aparelho monitor de glicemia, de forma asséptica, dentro das normas de segurança do paciente. O parâmetro de referência utilizado foi o preconizado pelas diretrizes da Sociedade Brasileira de Diabetes ${ }^{(10)}$.

Para a análise dos resultados, utilizou-se o software Stata versão 12 (StataCorp LP, CollegeStation, TX, USA). Inicialmente, os dados foram submetidos à análise exploratória e descritiva, sendo as variáveis categóricas expressas em frequência absoluta e percentual.

A normalidade dos dados foi verificada pelo teste de Kolmogorov-Smirnov. Os resultados obtidos pelo referido teste atenderam a pressuposição que os dados tenham distribuição normal. Contemplando ainda os pressupostos, foram realizadas análises de resíduos, os resultados revelaram não haver evidência de que a suposição de homocedasticidade foi violada ou que uma transformação da variável resposta ou das explicativas fosse necessária.

Posteriormente, verificou-se a associação entre as variáveis por meio da regressão linear simples com os testes $\mathrm{F}$ de Snedecor e $\mathrm{t}$ de Student, utilizando-se para avaliação dos resultados o nível de significância de $(p<0,05)$.

O desenvolvimento do estudo atendeu às normas nacionais e internacionais de ética em pesquisa envolvendo seres humanos, vigente na época de realização do estudo. O Comitê de Ética em Pesquisa em Seres Humanos do Setor de Ciências da Saúde aprovou-o sob parecer nº 172.965.

\section{RESULTADOS}

Do total de participantes, verificou-se que 63\% $(n=63)$ eram mulheres, com média de idade de 49,5 anos (DP=16,7 anos), idade mínima de 16 e máxima de 84 anos. Houve predomínio dos brancos ( $\mathrm{n}=82$; $82 \%)$, com ensino fundamental completo $(n=21 ; 21 \%)$, que recebiam mais de $R \$ 901,00$ por mês $(n=61$; $61 \%$ ) e casados ( $n=49 ; 49 \%$ ) (Tabela 1$)$. 
Tabela 1 - Distribuição absoluta e percentual dos fumantes quanto às variáveis sociodemográficas. Ponta Grossa, PR, Brasil, 2015

\begin{tabular}{|c|c|}
\hline Variável & n (\%) \\
\hline \multicolumn{2}{|l|}{ Sexo } \\
\hline Feminino & $63(63)$ \\
\hline Masculino & $37(37)$ \\
\hline \multicolumn{2}{|l|}{ Idade } \\
\hline 15-25 anos & $10(10)$ \\
\hline $26-35$ anos & $17(17)$ \\
\hline $36-45$ anos & $9(9)$ \\
\hline $46-55$ anos & $21(21)$ \\
\hline $56-65$ anos & $27(27)$ \\
\hline 66 ou mais & 16(16) \\
\hline \multicolumn{2}{|l|}{ Cor (autorreferida) } \\
\hline Branca & $82(82)$ \\
\hline Negra & $7(7)$ \\
\hline Parda & 11(11) \\
\hline \multicolumn{2}{|l|}{ Escolaridade } \\
\hline Analfabeto & $4(4)$ \\
\hline Fundamental Completo & $21(21)$ \\
\hline Médio Completo & $20(20)$ \\
\hline Superior Incompleto & $2(2)$ \\
\hline \multicolumn{2}{|l|}{ Renda } \\
\hline Sem Renda & $2(2)$ \\
\hline Entre $R \$ 301,00$ e 600,00 & $5(5)$ \\
\hline Entre $R \$ 601,00$ e 900,00 & $32(32)$ \\
\hline$>\mathrm{R} \$ 901,00$ & $61(61)$ \\
\hline \multicolumn{2}{|l|}{ Estado Civil } \\
\hline Casado & $49(49)$ \\
\hline Divorciado & 12(12) \\
\hline Solteiro & $21(21)$ \\
\hline Viúvo & $18(18)$ \\
\hline
\end{tabular}

O hábito de fumar iniciou-se, em média, aos 16,7 anos de idade, com idade mínima de 6 anos. O consumo médio diário de cigarro foi de 17,7 (DP=10,7), com no mínimo 1 cigarro e máximo de 60 cigarros por dia. O grau de dependência à nicotina identificado por meio da escala de Fagerström revelou que $11 \%(n=11)$ dos entrevistados apresentavam dependência muito baixa, $20 \%(n=20)$ dependência baixa, $13 \%(n=13)$ dependência média e 56\% $(n=56)$ grau de dependência elevado ou muito elevado.

Na Tabela 2, observa-se que o maior tempo médio de fumo foi no sexo feminino (32,92 anos), entre os viúvos (41,94 anos), com renda entre 301-600 reais (42 anos) e de cor autorreferida negra (41,57 anos). Os participantes com maior escolaridade (superior incompleto) tinham menor tempo médio de fumo 28,71 anos $(ß=-17,54 ; p<0,05)$. 
Tabela 2 - Média do tempo de tabagismo (em anos), coeficientes Beta da regressão linear simples e seus intervalos de confiança (IC95\%), segundo, variáveis sociodemográficas. Ponta Grossa, PR, Brasil, 2015

\begin{tabular}{|c|c|c|c|c|}
\hline Variáveis & $\begin{array}{l}\text { Médias de Tempo } \\
\text { de Tabagismo }\end{array}$ & Coeficiente Beta & IC 95\%* & $\begin{array}{l}\text { Valor } \\
\text { de } p^{* *}\end{array}$ \\
\hline \multicolumn{5}{|l|}{ Sexo } \\
\hline Masculino & 31,71 & 1,21 & $(98,44-6,02)$ & 0,74 \\
\hline Feminino & 32,92 & & & \\
\hline Idade & - & 0,9 & $(0,81-0,99)$ & 0,001 \\
\hline \multicolumn{5}{|l|}{ Estado Civil } \\
\hline Casado & 32,38 & - & & \\
\hline Separado & 40,75 & 8,37 & $(-1,57--18,32)$ & 0,098 \\
\hline Solteiro & 20,25 & $-12,13$ & $(-20,32--3,93)$ & 0,004 \\
\hline Viúvo & 41,94 & 9,56 & $(0,67-18,45)$ & 0,035 \\
\hline \multicolumn{5}{|l|}{ Escolaridade } \\
\hline Analfabeto & 46,25 & - & - & \\
\hline Fundamental Completo & 24,05 & $-22,19$ & $(-39,89--4,50)$ & 0,015 \\
\hline Médio Completo & 28,71 & $-17,54$ & $(-35,8-0,010)$ & 0,05 \\
\hline Superior incompleto & 15 & $-31,25$ & $(-67,21-4,71)$ & 0,088 \\
\hline \multicolumn{5}{|l|}{ Renda (R\$) } \\
\hline Nenhuma & 4,5 & - & - & - \\
\hline 301 a 600 & 42 & 37,5 & $(11,02-63,98)$ & 0,006 \\
\hline 601 a 900 & 39,06 & 34,56 & $(11.48-57.65)$ & 0,004 \\
\hline 901 e mais & 29,12 & 24,62 & $(1.86-47.37)$ & 0,036 \\
\hline \multicolumn{5}{|l|}{ Cor } \\
\hline Branca & 31,06 & - & - & - \\
\hline Negra & 41,57 & 10,51 & $(-2,70-3,72)$ & 0,118 \\
\hline Parda & 36,82 & 5,75 & $(-5,04-16.54)$ & 0,292 \\
\hline
\end{tabular}

No que se refere aos antecedentes familiares para doenças cardiovasculares, o maior tempo foi identificado no acidente vascular cerebral (40,75 anos), com colesterol aumentado (39,8 anos), que faziam uso de anti-hipertensivo (43,53 anos), que não realizavam exercício físico (32,82 anos) (Tabela 3).

Tabela 3 - Média do tempo de tabagismo (em anos), coeficientes Beta da regressão linear simples e seus intervalos de confiança (IC 95\%), segundo, variáveis clínicas. Ponta Grossa, PR, Brasil, 2015 (continua)

\begin{tabular}{lcccc} 
Variáveis & $\begin{array}{c}\text { Médias de Tempo } \\
\text { de Tabagismo }\end{array}$ & $\begin{array}{c}\text { Coeficiente } \\
\text { Beta }\end{array}$ & IC 95\%* & $\begin{array}{c}\text { Valor } \\
\text { de } \mathbf{~ p}^{* *}\end{array}$ \\
\hline Antecedentes familiares para doenças cardiovasculares & & - & - \\
\hline Não & 30,59 & 10,16 & $(-1,35-21,67)$ & 0,083 \\
\hline Acidente Vascular Cerebral & 40,75 & $-0,88$ & $(-9,83-8,07)$ & 0,846 \\
\hline Diabetes Mellitus & 29,71 & $-2,59$ & $(-13,52-8,33)$ & 0,639 \\
\hline Hipertensão Arterial & 28 & 8,07 & $(-2,61--18,76)$ & 0,137 \\
\hline Infarto Agudo do Miocárdio & 38,67 & & & - \\
\hline Colesterol elevado & & - & - & - \\
\hline Não & 29,31 & 10,49 & $(0,54-20,45)$ & $\mathbf{0 , 0 3 9}$ \\
\hline Sim & 39,8 & & & - \\
\hline Uso de anti-hipertensivo & & - & - & $\mathbf{0 , 0 0 1}$ \\
\hline Não & 26,97 & 16,56 & $(10,06-23,07)$ & \\
\hline Sim & 43,53 & & & \\
\hline
\end{tabular}




\begin{tabular}{lcccc}
\hline Exercício Físico & & & & \\
\hline Não & 32,82 & - & - & - \\
\hline Sim & 31,72 & $-1,09$ & $(-8,62--6,43)$ & 0,773 \\
\hline Grau de dependência à nicotina & & & & \\
\hline Muito baixa & 22,4 & - & - & - \\
\hline Baixa & 34,05 & 11,65 & $(-1,05-24,35)$ & 0,072 \\
\hline Média & 27,54 & 5,14 & $(-8,66-18,94)$ & 0,461 \\
\hline Elevada & 31,41 & 9,01 & $(-3,02-21,04)$ & 0,14 \\
\hline Muito elevada & 39,38 & 16,98 & $(4,63-29,32)$ & 0,008 \\
\hline Peso & - & $-0,19$ & $(-0,38--0,01)$ & $\mathbf{0 , 0 4 7}$ \\
\hline Circunferência abdominal & - & 0,19 & $(-0,04-0,44)$ & 0,1 \\
\hline Altura & - & $-43,78$ & $(-82,80--4.78)$ & $\mathbf{0 , 0 2 8}$ \\
\hline IMC & - & $-0,33$ & $(-0,88-0,22)$ & 0,232 \\
\hline Glicemia & - & 0,04 & $(-0,02-0,11)$ & 0,161
\end{tabular}

Constatou-se correlação estatística significativa entre maior tempo médio de fumo e grau de dependência à nicotina muito elevada $(B=16,98 ; p>0,008)$. Verificou-se associação entre o aumento do tempo de fumo e as variáveis peso $(ß=0,19 ; p<0,047)$ e altura $(ß=-43,78 ; p<0,028)$ (Tabela 3$)$.

\section{DISCUSSÃO}

No que se refere às variáveis sociodemográficas da amostra, os achados são semelhantes a outras investigações que avaliaram o perfil de usuários de tabaco, quanto ao predomínio do sexo feminino ${ }^{(11-13)}$, estado civil casado ${ }^{(11-12)}$ e escolaridade média de $8 \operatorname{anos}^{(11)}$.

Quanto ao início do hábito de fumar, identificou-se neste estudo que foi precoce - em média aos 16,7 anos de idade - e está em consonância com a realidade brasileira. Segundo o Instituto Nacional de Câncer (INCA) ${ }^{(4)}$, a idade média de experimentação de tabaco entre os jovens é de 16 anos de idade em ambos os sexos. Em pesquisa realizada no Brasil com 74.589 adolescentes de 15 a 17 anos, verificou-se prevalências mais elevadas de tabagismo quando comparados aos de 12 a 14 anos $^{(14)}$.

Autores destacam que o tabagismo é uma doença pediátrica, uma vez que cerca de $90 \%$ dos fumantes regulares iniciam antes dos 18 anos de idade ${ }^{(15)}$. Nesse contexto, identificar a idade de início de experimentação do fumo contribui para a determinação do tempo de exposição e fatores de risco para doenças, bem como direciona a equipe de saúde na condução de ações educativas antitabágicas direcionadas a grupos de vulneráveis.

O consumo médio diário de cigarros - 17,7 ( $\mathrm{DP}=10,7)$ - foi semelhante ao encontrado na literatura nacional e internacional ${ }^{(11,16)}$ e significativamente mais alto que a investigação transversal com adultos mexicanos $^{(13)}$, a qual encontrou uma média de 5 cigarros/dia. Contudo, comparações devem ser realizadas com cautela, tendo em vista que diferentes estratégias de aferição do consumo de cigarros são empregadas nas pesquisas. No Brasil, segundo o Relatório do Projeto ITC-Brasil ${ }^{(17)}$ o número médio de cigarros fumados diariamente é de 17 cigarros.

O número de cigarros consumidos está relacionado ao risco de morte por câncer de pulmão. De acordo com o INCA (5), indivíduos que fumam entre 1 a 14 cigarros/dia, 15 a 24 cigarros/dia e mais de 25 cigarros/dia têm, respectivamente, risco 8, 14 e 24 vezes maior de morte por este tipo de câncer do que pessoas que nunca fumaram. Por outro lado, a cessação do fumo reduz significativamente o risco de morte por causas associadas ao tabaco, aumentando em 9 anos a sobrevida média de uma população.

Os achados da presente pesquisa mostraram que houve predomínio dos usuários classificados nos níveis elevados e muito elevados de dependência à nicotina. Autores ${ }^{(18)}$ apontam que indivíduos com escores baixos no FTND estão mais propensos a abandonarem o hábito de fumar. Logo, supõe-se que os sujeitos pesquisados neste estudo tenham maior dificuldade de cessar o tabagismo e necessitariam 
de estratégias específicas para apoio à cessação.

A amostra dessa investigação foi predominantemente feminina, com maior tempo de tabagismo para essa população. Uma possível justificativa para tal achado pode ser atribuída ao fato da coleta de dados ter sido realizada nos domicílios, em horário que a maioria dos homens estavam no trabalho. Segundo a Pesquisa Nacional de Saúde de $2013^{(19)}$ os homens representam maior percentual de usuários do tabaco $(19,2 \%)$ em comparação com as mulheres $(11,2 \%)$. E, apesar da prevalência do tabagismo ser maior entre os homens ${ }^{(1-2)}$, autores constataram maior média de tempo de fumo no sexo feminino ${ }^{(20)}$.

O tabaco aumenta significativamente o risco para doenças cardiovasculares nas mulheres, com destaque para o Infarto Agudo do Miocárdio ${ }^{(2,5)}$. Do mesmo modo, para desenvolver diversos tipos de cânceres, em especial o de pulmão e brônquios que se configuram como a segunda neoplasia que mais causa óbito nas mulheres brasileiras ${ }^{(5)}$. Nesse contexto, ressalta-se a importância de ações de prevenção voltadas a esse grupo, que se enquadra no que mais procura ajuda para cessação de tabagismo.

A média de idade foi significativamente maior em comparação ao estudo transversal realizado no estado de Santa Catarina, com 707 indivíduos da comunidade na faixa etária entre 20 e 59 anos, o qual identificou média de idade de 38,7 anos ${ }^{(11)}$. Tal diferença pode ser explicada pelas características sociodemográficas e culturais da amostra. Neste estudo, constatou-se associação significativa da média de tempo de tabagismo com idade, do mesmo modo que pesquisa transversal de base populacional conduzida com 1.512 indivíduos adultos residentes nas zonas urbana e rural $(p=0,019)^{(12)}$.

Identificou-se que os viúvos apresentaram maiores médias de tempo de tabagismo. Tal resultado pode ser atribuído à situação de vulnerabilidade comum nos indivíduos divorciados, viúvos ou que moram sozinhos. Por outro lado, nos casados ou que vivem com companheiro, o apoio entre o casal pode favorecer o abandono do uso de tabaco ${ }^{(21)}$.

Entretanto, em pesquisa transversal de base populacional ${ }^{(22)}$ com 2.153 mulheres, constatou-se que aquelas em união estável são mais propensas a serem fumantes em comparação com as solteiras (OR $=2,49$; IC95\%: 1,12-5,53).

Observou-se maior tempo médio de tabagismo nos indivíduos com renda menor que um salário mínimo e com menor escolaridade. A prevalência de fumantes é inversamente proporcional à escolaridade e renda ${ }^{(23-24)}$. Do mesmo modo, autores ${ }^{(20)}$ ressaltam que a população sem ou com pouca instrução apresenta quase o dobro da prevalência de tabagismo daqueles com maior escolaridade. Dessa forma, a escolaridade constitui um fator de proteção, tanto no Brasil( ${ }^{(3,5,20)}$ quanto em outros países $^{(1,25)}$.

Compreende-se que a maior concentração do tabagismo nas populações de baixa renda e pouca escolaridade pode estar relacionada ao menor acesso à informação, percepção de autocuidado, qualidade de vida e acesso à medicina preventiva.

Os participantes de cor autorreferida negra apresentaram maior tempo de tabagismo. Tal resultado está em consonância com a literatura ${ }^{(3,20)}$, que aponta as pessoas declaradas da cor ou raça preta apresentam maior consumo de tabaco (17,8\%) em relação aos indivíduos brancos $(13,1 \%)$.

Relativo às variáveis clínicas, verificou-se maior tempo de tabagismo entre os participantes que possuíam colesterol elevado e utilizavam anti-hipertensivo. Isoladamente, o consumo de cigarro se configura como um grave problema de saúde pública e importante fator de risco para ocorrência das doenças crônicas não transmissíveis, e também como causa contribuinte para o surgimento de dislipidemia e hipertensão arterial.

Em clássico estudo sobre a mortalidade entre homens fumantes, autores ${ }^{(26)}$ verificaram que o tabagismo foi importante causa de morte por diversas condições crônicas. No presente estudo, a média de cigarros fumados/dia é elevada $(17,7)$. Tendo como referência os dados do estudo supracitado ${ }^{(26)}$, indivíduos que fumam entre 15 e 24 cigarros/dia, com idade menor que 45 anos, morrem oito vezes mais que os não fumantes por doença isquêmica do coração, mais de quatro vezes na idade entre 65 e 74 anos por degeneração do miocárdio, e 2,7 vezes a mais por trombose cerebral nesta mesma faixa etária. 
Entre os participantes, constatou-se alto índice de sobrepeso e obesidade, contudo, diferentemente da investigação transversal com estudantes de universidades públicas e privadas da cidade São LuísMA, não houve associação estatística significativa do tabagismo com IMC ( $p=0,010)$ e circunferência abdominal $(p=0,036)^{(27)}$.

Diante dos resultados apresentados, compreende-se a importância dos profissionais inseridos na ESF identificarem os fatores associados ao tabagismo nos usuários com ênfase em ações preventivas às complicações que dele advém em longo prazo.

As limitações deste estudo estão relacionadas ao delineamento de pesquisa transversal, que não possibilita verificar as relações de causa e efeito entre as variáveis ao longo do tempo. A pesquisa foi realizada com uma amostra por conveniência que, adicionada ao horário da visita domiciliar (realizada no período matutino e vespertino), pode ter evidenciado uma representatividade menor de população masculina na amostra.

No entanto, como a literatura é escassa sobre o tema nas Estratégias Saúde da Família, os resultados deste estudo são úteis ao planejamento local de saúde, e também para os municípios que têm alta cobertura de Saúde da Família e necessitam estar atentos ao cuidado longitudinal, para prevenir a ocorrência de doenças crônicas ou a agudização destas.

\section{CONSIDERAÇÕES FINAIS}

O estudo possibilitou identificar os fatores associados ao tabagismo em usuários da Estratégia Saúde da Família com destaque para idade, escolaridade, estado civil, colesterol elevado, uso de anithipertensivo, peso e altura. A identificação dos fatores associados ao tabagismo possibilita à equipe de saúde buscar estratégias para melhorar o cuidado da população da sua área de abrangência, abrindo portas para parceria entre ensino e serviço no encaminhamento dos tabagistas para programas de cessação do fumo.

Sugere-se, além da parceria com as instituições de ensino, que seja estabelecida uma rede de atenção ao tabagista, interligando os serviços de atenção primária com os demais níveis do sistema. Desse modo, a rede de cuidados pode atuar na prevenção da iniciação precoce deste hábito nocivo à saúde, e no cuidado aos sujeitos que já são dependentes do tabaco, com o objetivo de diminuir os fatores de risco modificáveis pela atenção à saúde e consequentemente reduzir a incidência de doenças crônicas não transmissíveis.

\section{REFERÊNCIAS}

1. World Health Organization (WHO). Report on the global tobacco epidemic, 2013. Enforcing bans on tobacco advertising, promotion and sponsorship. [Internet] Geneva: WHO; 2013 [acesso em 17 mai 2016]. Disponível: http://www.who.int/tobacco/global_report/2013/en/.

2. Ministério da Saúde (BR). Secretaria de Atenção à Saúde. Departamento de Atenção Básica. Estratégias para o cuidado da pessoa com doença crônica: o cuidado da pessoa tabagista. Brasília: Ministério da Saúde; 2015.

3. Instituto Brasileiro de Geografia e Estatística (IBGE). Pesquisa Nacional de Saúde 2013: percepção do estado de saúde, estilos de vida e doenças crônicas. [Internet] Rio de Janeiro: IBGE; 2014a [acesso em 10 fev 2016]. Disponível: ftp://ftp.ibge.gov.br/PNS/2013/pns2013.pdf.

4. Ministério da Saúde (BR). Instituto Nacional do Câncer (INCA). Plano de Implantação da Abordagem e Tratamento do Tabagismo na Rede SUS. [Internet] Rio de Janeiro: INCA; 2014a [acesso em 10 fev 2016]. Disponível: http://www2.inca.gov.br/wps/wcm/connect/3b4da40047eaabc088d4cd9ba9e4feaf/plano-de-implantacao-daabordagem-e-tratamento-do-tabagismo-na-rede-sus.pdf?MOD=AJPERES\&CACHEID=3b4da40047eaabc088d4 cd9ba9e4feaf.

5. Ministério da Saúde (BR). Instituto Nacional do Câncer (INCA). Coordenação de Prevenção e Vigilância. Estimativa 2014: incidência de câncer no Brasil. Brasília: INCA; 2014b. 
6. Araujo RB, Oliveira MS, Moraes JFD, Pedroso RS, Port F, de Castro MGT. Validação da versão brasileira do Questionnaire of Smoking Urges-Brief. Rev. psiquiatr. clín. [Internet] 2007;34(4) [acesso em 17 ago 2017]. Disponível: http://dx.doi.org/10.1590/S0101-60832007000400002.

7. World Health Organization (WHO). Obesity: preventing and managing the global epidemic. [Internet] 1998 [acesso em 1 jun 2016]. Disponível: http://www.who.int/nutrition/publications/obesity/WHO_TRS_894/en/.

8. American Diabetes Association (ADA). Diagnosis and classification of diabetes mellitus. Diabetes Care. [Internet] 2013;36(Suppl 1) [acesso em 14 mai 2016]. Disponível: https://doi.org/10.2337/dc13-S067.

9. Sociedade Brasileira de Cardiologia. Sociedade Brasileira de Hipertensão. Sociedade Brasileira de Nefrologia. VII Diretrizes Brasileiras de Hipertensão. Arq Bras Cardiol. [Internet] 2016;107(3 Suppl 3) [acesso em 14 mai 2016]. Disponível: http://publicacoes.cardiol.br/2014/diretrizes/2016/05_HIPERTENSAO_ARTERIAL.pdf.

10. Sociedade Brasileira de Diabetes (SBD). Diretrizes da Sociedade Brasileira de Diabetes: 2015-2016. São Paulo: AC Farmacêutica; 2016.

11. Bortoluzzi MC, Kehrig RT, Loguercio AD, Traebert JL. Prevalence and tobacco user profile in adult population in the South of Brazil (Joaçaba, SC). Ciênc. saúde coletiva. [Internet] 2011;16(3) [acesso em 14 mai 2016]. Disponível: http://dx.doi.org/10.1590/S1413-81232011000300029.

12. Martinelli PM, Lopes CM, Muniz PT, de Souza OF. Smoking in adults in the municipality of Rio Branco, Acre, Brazil: a population-based study. Rev. bras. epidemiol. [Internet] 2014;17(4) [acesso em 14 mai 2016]. Disponível: http://dx.doi.org/10.1590/1809-4503201400040016.

13. Ruiz-Juan F, Isorna-Folgar M, Vaquero-Cristóba R, Ruiz-Risueño J. Consumo de tabaco en adultos de Monterrey: relación com actividad físico-deportiva y família. Nutr. Hosp. [Internet] 2016;33(2) [acesso em 14 mai 2016]. Disponível: http://dx.doi.org/10.20960/nh.523.

14. Figueiredo VC, Szklo AS, Costa LC, Kuschnir MCC, da Silva TLN, Bloch KV, et al. ERICA: smoking prevalence in Brazilian adolescents. Rev. Saúde Pública. [Internet] 2016;50(Suppl 1) [acesso em 14 mai 2016]. Disponível: http:// dx.doi.org/10.1590/S01518-8787.2016050006741.

15. da Silva ST, Martins MC, de Faria FR, Cotta RMM. Combate ao Tabagismo no Brasil: a importância estratégica das ações governamentais. Ciênc. saúde coletiva. [Internet] 2014;19(2) [acesso em 5 ago 2016]. Disponível: http:// dx.doi.org/10.1590/1413-81232014192.19802012.

16. Li S, Meng L, Chiolero A, Ma C, Xi B. Trends in smoking prevalence and attributable mortality in China, 19912011. Preventive Medicine. [Internet] 2016;(93) [acesso em 12 ago 2016]. Disponível: http://dx.doi.org/10.1016/j. ypmed.2016.09.027.

17. Ministério da Saúde (BR). Instituto Nacional do Câncer (INCA). Relatório Projeto ITC. Relatório do Projeto ITC-Brasil. Resultados das Ondas 1 e 2 da Pesquisa (2009-2013) - maio 2014: Sumário executivo. [Internet] Rio de Janeiro: INCA; 2014c [acesso em 10 fev 2016]. Disponível: http://actbr.org.br/uploads/conteudo/913_ITC_ sumario.pdf.

18. Pérez-Padilla R, Stelmach R, Soto-Quiroz M, Cruz AA. Combate a doenças respiratórias: esforços divididos levam ao enfraquecimento. J. bras. pneumol. [Internet] 2014;40(3) [acesso em 12 ago 2016]. Disponível: http:// dx.doi.org/10.1590/S1806-37132014000300001.

19. Instituto Brasileiro de Geografia e Estatística (IBGE). Pesquisa Nacional de Saúde 2013: percepção do estado de saúde, estilos de vida e doenças crônicas: Brasil, grandes regiões e unidades da federação [Internet]. Rio de Janeiro: IBGE; 2014b [acesso em 15 fev 2016]. Disponível: http://biblioteca.ibge.gov.br/visualizacao/livros/ liv91110.pdf.

20. Malta DC, Oliveira TP, Luz M, Stopa SR, da Silva Junior JB, dos Reis AAC. Smoking trend indicators in Brazilian capitals, 2006-2013. Ciênc. saúde coletiva. [Internet] 2015;20(3) [acesso em 10 fev 2016]. Disponível: http://dx.doi. org/10.1590/1413-81232015203.15232014.

21. de Jesus MCP, da Silva MH, Cordeiro SM, Korchmar E, Zampier VSB, Merighi MAB. Understanding unsuccessful attempts to quit smoking: a social phenomenology approach. Rev. esc. enferm. USP. [Internet] 2016;50(1) [acesso em 2 set 2016]. Disponível: http://dx.doi.org/10.1590/S0080-623420160000100010. 
22. Scarinci IC, Bittencourt L, Person S, Cruz RC, Moysés ST. Prevalência do uso de produtos derivados do tabaco e fatores associados em mulheres no Paraná, Brasil. Cad. Saúde Pública. [Internet] 2012;28(8) [acesso em 22 out 2016]. Disponível: http://dx.doi.org/10.1590/S0102-311X2012000800004.

23. Ministério da Saúde (BR). Agência Nacional de Saúde Suplementar. Saúde Suplementar: vigilância de fatores de risco e proteção para doenças crônicas por inquérito telefônico.Brasília: Ministério da Saúde; 2017.

24. Malta DC, Oliveira TP, Vieira ML, Almeida L, Szwarcwald CL. Use of tobacco and exposure to tobacco smoke in Brazil: results from the National Health Survey 2013. Epidemiol. Serv. Saúde. [Internet] 2015;24(2) [acesso em 2 jun 2016]. Disponível: http://dx.doi.org/10.5123/S1679-49742015000200006.

25. Giovino GA, Mirza SA, Samet JM, Gupta PC, Jarvis MJ, Bhala N, et al. Tobacco use in 3 billion individuals from 16 countries: an analysis of nationally representative cross-sectional household surveys. The Lancet. [Internet] 2012;380(9842) [acesso em 15 mar 2016]. Disponível: http://dx.doi.org/10.1016/S0140-6736(12)61085-X.

26. Doll R, Peto R. Mortality in relation to smoking: 20 years'observations on male British doctors. Br Med J. [Internet] 1976;2(6051) [acesso em 22 set 2016]. Disponível: https://www.ncbi.nlm.nih.gov/pmc/articles/ PMC1690096.

27. Carvalho CA, Fonseca PCA, Barbosa JB, Machado SP, Santos AM, Silva AAM. The association between cardiovascular risk factors and anthropometric obesity indicators in university students in São Luís in the State of Maranhão, Brazil. Ciênc. saúde coletiva. [Internet] 2015;20(2) [acesso em 14 mai 2016]. Disponível: http://dx.doi. org/10.1590/1413-81232015202.02342014. 